\title{
LOCAL EXISTENCE FOR A GENERAL MODEL OF SIZE-DEPENDENT POPULATION DYNAMICS
}

\author{
NOBUYUKI KATO AND HIROYUKI TORIKATA
}

\begin{abstract}
We shall investigate a size structured population dynamics with aging and birth functions having general forms. The growth rate we deal with depends not only on the size but also on time. We show the existence of a local solution and continuous dependence on the initial data, which shows the uniqueness of the solution as well.
\end{abstract}

\section{INTRODUCTION}

We are interested in a size structured population model with the growth rate depending on the individual's size and time. There has been many investigations where the growth rate depends on the size. See, for example, [2; Chap. 10], [3] and the references therein. Recently, A. Calsina and J. Saldaña [1] have studied the case where the growth rate depends on the size as well as the total population at each time. They have the model of plants in forests or plantations in their mind.

We are also motivated by the population model of the forest growth etc. In this case, the growth rate may be influenced by the environment such as light, temperature, and nutrients. These may change with time. It is also reasonable to think that the growth rate varies with the individual's size of plants because the amount of light they capture may depend on it. From these points of view, it is natural to consider the growth rate depending on the size and time.

1991 Mathematics Subject Classification. 92D25.

Key words and phrases. Population dynamics, size-dependent, local existence of solutions, uniqueness of solutions.

Received: April 30, 1997. 
In this paper we study the following initial boundary value problem with nonlocal boundary condition:

$$
\left\{\begin{array}{l}
u_{t}+(V(x, t) u)_{x}=G(u(\cdot, t))(x), \quad x \in[0, l), a \leq t \leq T, \\
V(0, t) u(0, t)=C(t)+F(u(\cdot, t)), \quad a \leq t \leq T, \\
u(x, a)=u_{a}(x), \quad x \in[0, l) .
\end{array}\right.
$$

Here $a \geq 0, l \in(0, \infty]$ is the maximum size, $F$ and $G$ are given mappings corresponding to birth and aging functions respectively. The function $V$ is the growth rate function depending on the size $x$ and time $t$ and the function $C$ represents the inflow of zero-size individuals from an external source such as seeds carried by the wind or placed in a plantation. The unknown function $u(x, t)$ stands for the density with respect to size $x$ of a population at time $t$. So the integral $\int_{x_{1}}^{x_{2}} u(x, t) d x$ represents the number of individuals with size between $x_{1}$ and $x_{2}$ at time $t$. The equation (SDP) is closely related to the age-dependent population dynamics developed by $\mathrm{G}$. Webb [4]. Indeed, from the mathematical point of view, the particular case $V(x, t) \equiv 1$ is nothing but the age-dependent case.

Our objective is to show the existence of a unique local solution and continuous dependence of the solution on the initial data. The results extend [4, Propositions 2.2 and 2.3] and partially [1, Theorems 1 and 2]. The birth and aging functions treated in [1] are of Gurtin-MacCamy type, i.e.,

$$
F(u(\cdot, t))=\int_{0}^{l} \beta(x, P(t)) u(x, t) d x, \quad G(u(\cdot, t))(x)=-m(x, P(t)) u(x, t)
$$

where $P(t)=\int_{0}^{l} u(x, t) d x$ is the total population at time $t$, and this is essential for their arguments. We handle more general birth and aging functions which are the same as in [4]. In [1], the growth rate function $V$ depends on the size and the total population $P(t)$, while we deal with $V$ depending on the size and time.

The paper is organized as follows. In Section 2 we state our assumptions and main results (Theorems 2.1 and 2.2). We prepare some lemmas in Section 3 and the proofs of the theorems are established in Sections 4 and 5 .

\section{LOCAL EXISTENCE AND UNIQUENESS}

In this section we state our main theorems concerning the existence of a unique local solution to (SDP) and the continuous dependence on the initial data. At first, we introduce some notations.

Let $L^{1}:=L^{1}\left(0, l ; \mathbb{R}^{n}\right)$ be the Banach space of Lebesgue integrable functions from $[0, l)$ to $\mathbb{R}^{n}$ with norm $\|f\|_{L^{1}}:=\int_{0}^{l}|f(x)| d x$ for $f \in L^{1}$, where $|\cdot|$ denotes the norm of $\mathbb{R}^{n}$. For $T>a$ we set $L_{a, T}:=C\left([a, T] ; L^{1}\right)$, the Banach space of $L^{1}$-valued continuous functions on $[a, T]$ with the supremum 
norm $\|u\|_{L_{a, T}}:=\sup _{a \leq t \leq T}\|u(t)\|_{L^{1}}$ for $u \in L_{a, T}$. Note that each element of $L_{a, T}$ is identified with an element of $L^{1}\left((0, l) \times(a, T) ; \mathbb{R}^{n}\right)$ by the relation $[u(t)](x)=u(x, t)$ for $a \leq t \leq T$, a.e. $0<x<l$. See [4, Lemma 2.1].

We assume the following hypotheses.

(F) $F: L^{1} \rightarrow \mathbb{R}^{n}$ is locally Lipschitz in the sense that there is an increasing function $c_{1}:[0, \infty) \rightarrow[0, \infty)$ such that $\left|F\left(\phi_{1}\right)-F\left(\phi_{2}\right)\right| \leq$ $c_{1}(r)\left\|\phi_{1}-\phi_{2}\right\|_{L^{1}}$ for all $\phi_{1}, \phi_{2} \in L^{1}$ with $\left\|\phi_{1}\right\|_{L^{1}},\left\|\phi_{2}\right\|_{L^{1}} \leq r$.

(G) $G: L^{1} \rightarrow L^{1}$ is locally Lipschitz, i.e., there is an increasing function $c_{2}:[0, \infty) \rightarrow[0, \infty)$ such that $\left\|G\left(\phi_{1}\right)-G\left(\phi_{2}\right)\right\|_{L^{1}} \leq c_{2}(r)\left\|\phi_{1}-\phi_{2}\right\|_{L^{1}}$ for all $\phi_{1}, \phi_{2} \in L^{1}$ with $\left\|\phi_{1}\right\|_{L^{1}},\left\|\phi_{2}\right\|_{L^{1}} \leq r$.

(V) $V:[0, l) \times[0, T] \rightarrow(0, \infty)$ is a bounded function with upper bound $V_{0}>0, V(x, t)$ is Lipschitz continuous with respect to $x$ uniformly for $t$, i.e., there is a constant $L_{V}>0$ such that

$$
\left|V\left(x_{1}, t\right)-V\left(x_{2}, t\right)\right| \leq L_{V}\left|x_{1}-x_{2}\right|, \quad \forall x_{1}, x_{2} \in[0, l), t \in[0, T],
$$

and the mapping $t \mapsto V(x, t)$ is continuous for each $x \in[0, l)$. Further, if $l<\infty$, then $V(l, \cdot)=0$.

(C) $C:[0, T] \rightarrow \mathbb{R}^{n}$ is a continuous function.

We define the characteristic curve $\varphi\left(t ; t_{0}, x_{0}\right)$ through $\left(x_{0}, t_{0}\right) \in[0, l) \times$ $[0, T]$ by the solution of the differential equation

$$
\left\{\begin{array}{l}
x^{\prime}(t)=V(x(t), t), \quad t \in\left[t_{0}, T\right] \\
x\left(t_{0}\right)=x_{0} \in[0, l) .
\end{array}\right.
$$

Since the function $V$ is Lipschitz continuous as assumed in $(\mathrm{V})$, it is well known that there exists a unique solution $x(t)=\varphi\left(t ; t_{0}, x_{0}\right)$ of $(2.1)$ on $\left[t_{0}, T\right]$.

Let $z_{a}(t):=\varphi(t ; a, 0)$ denote the characteristic through $(0, a)$ in the $(x, t)$ plane. In particular, the curve $z_{0}(t)$ is the trajectory in the $(x, t)$-plane of the newborn individuals at $t=0$ and it separates the trajectories of the individuals that were present at the initial time $t=0$ from the trajectories of those individuals born after the initial time.

For $(x, t) \in[0, l) \times[0, T]$ such that $x<z_{0}(t)$, define $\tau:=\tau(t, x)$ implicitly by the relation

$$
\varphi(t ; \tau, 0)=x, \quad \text { or equivalently, } \quad \varphi(\tau ; t, x)=0
$$

i.e. $\tau$ is the initial time of the characteristic through $(x, t)$. And then define $\tau_{a}^{*}$ by

$$
\tau_{a}^{*}\left(t_{0}, x_{0}\right)= \begin{cases}\tau\left(t_{0}, x_{0}\right) & \text { for } x_{0}<z_{a}\left(t_{0}\right) \\ a & \text { for } x_{0} \geq z_{a}\left(t_{0}\right)\end{cases}
$$


It is obvious that the solution $x(t)=\varphi\left(t ; t_{0}, x_{0}\right)$ of $(2.1)$ can be extended on $\left[\tau_{a}^{*}\left(t_{0}, x_{0}\right), T\right]$ and $\varphi\left(t ; t_{0}, x_{0}\right)$ satisfies the integral equation

$$
\varphi\left(t ; t_{0}, x_{0}\right)=x_{0}+\int_{t_{0}}^{t} V\left(\varphi\left(\sigma ; t_{0}, x_{0}\right), \sigma\right) d \sigma \quad \text { for } t \in\left[\tau_{a}^{*}\left(t_{0}, x_{0}\right), T\right]
$$

Note also that $x(t)=\varphi\left(t ; t_{0}, x_{0}\right)$ satisfies $0 \leq x(t)<l$ for every $t \in$ $\left[\tau_{a}^{*}\left(t_{0}, x_{0}\right), T\right]$ provided $x_{0} \in[0, l)$, and if $l<\infty$ and $x_{0}=l$, then $x(t) \equiv l$.

With the characteristics $\varphi$ we define a solution of (SDP) as follows.

Definition 2.1. A function $u \in L_{a, T}$ is called a solution of (SDP) if $u$ satisfies

$$
\begin{aligned}
& u(x, t)= \\
& \begin{cases}\frac{\tilde{F}(\tau, u(\cdot, \tau))}{V(0, \tau)}+\int_{\tau}^{t} \tilde{G}(s, u(\cdot, s))(\varphi(s ; \tau, 0)) d s & \text { a.e. } x \in\left(0, z_{a}(t)\right), \\
u_{a}(\varphi(a ; t, x))+\int_{a}^{t} \tilde{G}(s, u(\cdot, s))(\varphi(s ; t, x)) d s & \text { a.e. } x \in\left(z_{a}(t), l\right),\end{cases}
\end{aligned}
$$

where $\tau:=\tau(t, x)$ is given by $(2.2), \tilde{F}(t, u(\cdot, t))$ and $\tilde{G}(t, u(\cdot, t))(x)$ are given by

$$
\begin{gathered}
\tilde{F}(t, u(\cdot, t)):=C(t)+F(u(\cdot, t)), \quad \forall t \in[a, T], \\
\tilde{G}(t, u(\cdot, t))(x):= \\
G(u(\cdot, t))(x)-V_{x}(x, t) u(x, t), \quad \forall t \in[a, T], \text { a.e. } x \in(0, l) .
\end{gathered}
$$

Remark 2.1. The above definition is the analogue of the age-dependent case $[4,(1.49)]$. Note that if $u(x, t)$ satisfies (SDP) in a strong sense, then it is easily seen that $u$ satisfies (2.5).

Our main results are the following two theorems.

Theorem 2.1. Let $(\mathrm{F}),(\mathrm{G}),(\mathrm{V})$, and $(\mathrm{C})$ hold and let $r>0$. Then there exists a $\delta>0$ such that for $u_{a} \in L^{1}$ satisfying $\left\|u_{a}\right\|_{L^{1}} \leq r$, there exists the unique solution $u \in L_{a, T}$ of (SDP) on $[a, T]$ with $T=a+\delta$ (in the sense of Definition 2.1).

Theorem 2.2. Suppose (F), (G), (V), and (C) hold and let $T>a$ and $r>0$. Let $u, \hat{u} \in L_{a, T}$ be the solutions of (SDP) with initial values $u_{a}$, $\hat{u}_{a} \in L^{1}$ respectively satisfying $\|u\|_{L_{a, T}},\|\hat{u}\|_{L_{a, T}} \leq r$. Then we have the following estimate:

$\|u(\cdot, t)-\hat{u}(\cdot, t)\|_{L^{1}} \leq \exp \left[\left(c_{1}(r)+c_{2}(r)+2 L_{V}\right)(t-a)\right]\left\|u_{a}-\hat{u}_{a}\right\|_{L^{1}}, \quad a \leq t \leq T$.

Remark 2.2. Theorem 2.2 shows the continuous dependence of the solution on the initial data as well as the uniqueness of the solution for the unitial data as long as the solution exists. 


\section{Properties of Characteristic CURVES}

In this section we collect some properties of the characteristic curves defined by (2.1) (or (2.4)) in the previous section. Before that, let us begin with the well-known lemma.

Lemma 3.1. (Gronwall's lemma) Let $f$, a, and $g$ be nonnegative continuous functions.

(i) If $f(t) \leq a(t)+\int_{s}^{t} g(\sigma) f(\sigma) d \sigma$ for $t \geq s$, then

$$
f(t) \leq a(t)+\int_{s}^{t} g(\sigma) a(\sigma) \exp \left(\int_{\sigma}^{t} g(\xi) d \xi\right) d \sigma \quad \text { for } t \geq s
$$

(ii) If $f(t) \leq a(t)+\int_{t}^{s} g(\sigma) f(\sigma) d \sigma$ for $t \leq s$, then

$$
f(t) \leq a(t)+\int_{t}^{s} g(\sigma) a(\sigma) \exp \left(\int_{t}^{\sigma} g(\xi) d \xi\right) d \sigma \quad \text { for } t \leq s
$$

The case (i) is the standard one. The case (ii) is less familiar but we omit the proof since it is quite similar.

Some properties of the characteristic curves $\varphi$ are given as

Lemma 3.2. Let $\varphi$ be the characteristic curves defined by the solution of $(2.1)$.

(i) For any $t_{0} \in[0, T], x_{0} \in[0, l)$, the mapping $t \mapsto \varphi\left(t ; t_{0}, x_{0}\right)$ is increasing and Lipschitz continuous on $\left[\tau_{0}^{*}\left(t_{0}, x_{0}\right), T\right]$ where $\tau_{0}^{*}\left(t_{0}, x_{0}\right)$ is defined by (2.3).

(ii) For any $t_{1} \in[0, T], x_{1} \in[0, l)$, the mapping $s \mapsto \varphi\left(t_{1} ; s, x_{1}\right)$ is decreasing and Lipschitz continuous on $\left[0, \sigma_{1}\right]$ where $\sigma_{1}:=\sigma\left(t_{1}, x_{1}\right)$ is defined implicitly by $\varphi\left(\sigma_{1} ; t_{1}, 0\right)=x_{1}$ if $t_{1}<\tau\left(T, x_{1}\right)$ and $\sigma_{1}=T$ if $t_{1} \geq \tau\left(T, x_{1}\right)$.

(iii) For any $t, t_{0} \in[0, T]$, the mapping $x \mapsto \varphi\left(t ; t_{0}, x\right)$ is increasing and Lipschitz continuous on $\left[\chi\left(t, t_{0}\right), l\right)$ where

$$
\chi\left(t, t_{0}\right)= \begin{cases}0 & \text { if } t \geq t_{0} \\ \varphi\left(t_{0} ; t, 0\right) & \text { if } t<t_{0} .\end{cases}
$$

Proof. (i) Since $V$ is positive, it is easily seen from (2.4) that the mapping $t \mapsto \varphi\left(t ; t_{0}, x_{0}\right)$ is increasing. For $t, \hat{t} \in\left[\tau_{0}^{*}\left(t_{0}, x_{0}\right), T\right]$, by $(2.4)$ and $(\mathrm{V})$, we obtain

$$
\left|\varphi\left(t ; t_{0}, x_{0}\right)-\varphi\left(\hat{t} ; t_{0}, x_{0}\right)\right|=\left|\int_{\hat{t}}^{t} V\left(\varphi\left(\sigma ; t_{0}, x_{0}\right), \sigma\right) d \sigma\right| \leq V_{0}|t-\hat{t}| .
$$


(ii) It is obvious that the mapping $s \mapsto \varphi\left(t_{1} ; s, x_{1}\right)$ is decrasing. For $s$, $\hat{s} \in\left[0, \sigma_{1}\right]$, by $(2.4)$ and $(\mathrm{V})$, we get

$$
\begin{aligned}
& \left|\varphi\left(t_{1} ; s, x_{1}\right)-\varphi\left(t_{1} ; \hat{s}, x_{1}\right)\right| \\
& \quad=\left|\int_{s}^{t_{1}} V\left(\varphi\left(\xi ; s, x_{1}\right), \xi\right) d \xi-\int_{\hat{s}}^{t_{1}} V\left(\varphi\left(\xi ; \hat{s}, x_{1}\right), \xi\right) d \xi\right| \\
& \quad \leq\left|\int_{s}^{t_{1}}\right| V\left(\varphi\left(\xi ; s, x_{1}\right), \xi\right)-V\left(\varphi\left(\xi ; \hat{s}, x_{1}\right), \xi\right)|d \xi|+\left|\int_{\hat{s}}^{s} V\left(\varphi\left(\xi ; \hat{s}, x_{1}\right), \xi\right) d \xi\right| \\
& \quad \leq L_{V}\left|\int_{s}^{t_{1}}\right| \varphi\left(\xi ; s, x_{1}\right)-\varphi\left(\xi ; \hat{s}, x_{1}\right)|d \xi|+V_{0}|s-\hat{s}| \\
& \quad= \begin{cases}L_{V} \int_{s}^{t_{1}}\left|\varphi\left(\xi ; s, x_{1}\right)-\varphi\left(\xi ; \hat{s}, x_{1}\right)\right| d \xi+V_{0}|s-\hat{s}| & \text { if } t_{1} \geq s, \\
L_{V} \int_{t_{1}}^{s}\left|\varphi\left(\xi ; s, x_{1}\right)-\varphi\left(\xi ; \hat{s}, x_{1}\right)\right| d \xi+V_{0}|s-\hat{s}| & \text { if } t_{1}<s .\end{cases}
\end{aligned}
$$

By Gronwall's lemma (Lemma 3.1), we have

$$
\left|\varphi\left(t_{1} ; s, x_{1}\right)-\varphi\left(t_{1} ; \hat{s}, x_{1}\right)\right| \leq V_{0}|s-\hat{s}| e^{L_{V} T}
$$

(iii) It is easily verified that the mapping $x \mapsto \varphi\left(t ; t_{0}, x\right)$ is increasing. If $x, \hat{x} \in\left[\chi\left(t, t_{0}\right), l\right)$, then we have

$$
\begin{aligned}
& \left|\varphi\left(t ; t_{0}, x\right)-\varphi\left(t ; t_{0}, \hat{x}\right)\right| \\
& \quad \leq|x-\hat{x}|+\left|\int_{t_{0}}^{t}\right| V\left(\varphi\left(\sigma ; t_{0}, x\right), \sigma\right)-V\left(\varphi\left(\sigma ; t_{0}, \hat{x}\right), \sigma\right)|d \sigma| \\
& \quad \leq|x-\hat{x}|+L_{V}\left|\int_{t_{0}}^{t}\right| \varphi\left(\sigma ; t_{0}, x\right)-\varphi\left(\sigma ; t_{0}, \hat{x}\right)|d \sigma| \\
& \quad= \begin{cases}|x-\hat{x}|+L_{V} \int_{t_{0}}^{t}\left|\varphi\left(\sigma ; t_{0}, x\right)-\varphi\left(\sigma ; t_{0}, \hat{x}\right)\right| d \sigma & \text { if } t \geq t_{0}, \\
|x-\hat{x}|+L_{V} \int_{t}^{t_{0}}\left|\varphi\left(\sigma ; t_{0}, x\right)-\varphi\left(\sigma ; t_{0}, \hat{x}\right)\right| d \sigma & \text { if } t<t_{0} .\end{cases}
\end{aligned}
$$

By Gronwall's lemma (Lemma 3.1), we obtain

$$
\left|\varphi\left(t ; t_{0}, x\right)-\varphi\left(t ; t_{0}, \hat{x}\right)\right| \leq|x-\hat{x}| e^{L_{V} T}
$$

This completes the proof.

The function $\tau$ defined by (2.2) has the following properties.

Lemma 3.3. (i) For any $t \in[0, T]$, put $\tau_{t}(x):=\tau(t, x)$. Then $\tau_{t}:\left[0, z_{0}(t)\right]$ $\rightarrow[0, t]$ is continuous, decreasing and onto, and hence invertible. The inverse function $\tau_{t}^{-1}(\cdot)$ is continuous from $[0, t]$ onto $\left[0, z_{0}(t)\right]$. Furthermore,

$$
\tau_{\hat{t}}^{-1}(s) \rightarrow \tau_{t}^{-1}(s) \quad \text { as } \hat{t} \rightarrow t \text { for each } s \in[0, t] .
$$


(ii) For each $x \in[0, l)$, the mapping $t \mapsto \tau(t, x)$ is increasing, and the mapping $(t, x) \mapsto \tau(t, x)$ is continuous on the region $U:=\{(t, x) \in[0, T] \times$ $\left.[0, l) \mid x<z_{0}(t)\right\}$.

Proof. (i) (Decreasing) For $x_{i} \in\left[0, z_{0}(t)\right]$, put $\tau_{i}:=\tau\left(t, x_{i}\right)(i=1,2)$. We will show that $\tau_{1}<\tau_{2}$ implies $x_{1}>x_{2}$. Suppose for contradiction that $x_{1} \leq x_{2}$. Since $x_{1}=\varphi\left(t ; \tau_{1}, 0\right)=\varphi\left(t ; \tau_{2}, \varphi\left(\tau_{2} ; \tau_{1}, 0\right)\right) \leq x_{2}=\varphi\left(t ; \tau_{2}, 0\right)$, there exists $t^{*} \in\left[\tau_{2}, t\right]$ such that

$$
\varphi\left(t^{*} ; \tau_{2}, \varphi\left(\tau_{2} ; \tau_{1}, 0\right)\right)=\varphi\left(t^{*} ; \tau_{2}, 0\right)\left(=: x_{0}\right) .
$$

This contradicts the fact that the initial value problem

$$
x^{\prime}(s)=V(x(s), s), \tau_{2}<s<t^{*}, \quad x\left(t^{*}\right)=x_{0}
$$

has a unique solution. Hence $x_{1} \geq x_{2}$, and so $\tau_{t}(\cdot)$ is shown to be decreasing.

(Onto) For any $s \in[0, t]$, putting $x:=\varphi(t ; s, 0)$, it is clear that $\tau_{t}(x)=s$. This shows that $\tau_{t}$ is onto.

(Continuity) For $x_{0} \in\left[0, z_{0}(t)\right]$, we put $\tau_{0}:=\tau_{t}\left(x_{0}\right)$. If $x_{0} \in\left(0, z_{0}(t)\right)$, then $0<\tau_{0}<t$. For any $\varepsilon>0$ such that $0<\tau_{0}-\varepsilon<\tau_{0}<\tau_{0}+\varepsilon<t$, we have

$$
\varphi\left(t ; \tau_{0}-\varepsilon, 0\right)>\varphi\left(t ; \tau_{0}, 0\right)=x_{0}>\varphi\left(t ; \tau_{0}+\varepsilon, 0\right) .
$$

Taking $\delta>0$ as $\delta:=\min \left\{\varphi\left(t ; \tau_{0}-\varepsilon, 0\right)-\varphi\left(t ; \tau_{0}, 0\right), \varphi\left(t ; \tau_{0}, 0\right)-\varphi\left(t ; \tau_{0}+\varepsilon, 0\right)\right\}$, it turns out that $\left|x-x_{0}\right|<\delta$ implies $\left|\tau_{t}(x)-\tau_{0}\right|<\varepsilon$. When $x_{0}=z_{0}(t)$ or $x_{0}=0$, one can observe that $\tau_{t}(\cdot)$ is right or left continuous, respectively, by the same fashion.

(Continuity of $\tau_{t}^{-1}$ ) For any $s_{0} \in[0, t]$, we put $x_{0}:=\tau_{t}^{-1}\left(s_{0}\right)=\varphi\left(t ; s_{0}, 0\right)$. If $s_{0} \in(0, t)$, then $0<x_{0}<z_{0}(t)$. Given $\varepsilon>0$ such that $0<x_{0}-\varepsilon<x_{0}<$ $x_{0}+\varepsilon<z_{0}(t)$, since $\tau_{t}$ is decreasing,

$$
\tau_{t}\left(x_{0}+\varepsilon\right)<\tau_{t}\left(x_{0}\right)=s_{0}<\tau_{t}\left(x_{0}-\varepsilon\right) .
$$

Taking $\delta>0$ as $\delta:=\min \left\{\tau_{t}\left(x_{0}-\varepsilon\right)-\tau_{t}\left(x_{0}\right), \tau_{t}\left(x_{0}\right)-\tau_{t}\left(x_{0}+\varepsilon\right)\right\}$, it is shown that $\left|s-s_{0}\right|<\delta$ implies $\left|\tau_{t}^{-1}(s)-\tau_{t}^{-1}\left(s_{0}\right)\right|=\left|\varphi(t ; s, 0)-\varphi\left(t ; s_{0}, 0\right)\right|<\varepsilon$. When $s_{0}=0$ or $s_{0}=t$, it is shown that $\tau^{-1}(\cdot)$ is right or left continuous respectively by the same way.

(Proof of (3.2)) We put $x:=\tau_{t}^{-1}(s)$. If $s \in(0, t)$, then $\varphi_{*}(t, T, x)<x<$ $z_{0}(t)$ where

$$
\varphi_{*}(t, T, x)= \begin{cases}\varphi(t ; T, x) & \text { if } t>\tau(T, x), \\ 0 & \text { if } t \leq \tau(T, x) .\end{cases}
$$

For any $\varepsilon>0$ such that $\varphi_{*}(t, T, x)<x-\varepsilon<x<x+\varepsilon<z_{0}(t)$, there exist $t_{1}, t_{2} \in(s, T)$ such that $x=\varphi\left(t_{1} ; t, x+\varepsilon\right)=\varphi\left(t_{2} ; t, x-\varepsilon\right)$. Taking $\delta>0$ as $\delta:=\min \left\{t-t_{1}, t_{2}-t\right\}$, it turns out that $|\hat{t}-t|<\delta$ implies $\left|\tau_{\hat{t}}^{-1}(s)-\tau_{t}^{-1}(s)\right|<\varepsilon$. When $s=0$ or $s=t$, the right or left continuity is verified respectively by the same way. 
(ii) It is easily seen that $t \mapsto \tau(t, x)$ is increasing. To prove the continuity of $\tau(t, x)$, first we observe that for any $t \in[0, T]$ and $x \in\left[0, z_{0}(t)\right), \tau(\hat{t}, x) \rightarrow$ $\tau(t, x)$ as $\hat{t} \rightarrow t$.

If $x \in\left(0, z_{0}(t)\right)$, then $0<\tau(t, x)<\tau_{*}(t, T, x)$ where

$$
\tau_{*}(t, T, x)= \begin{cases}\tau(T, x) & \text { if } t>\tau(T, x) \\ 0 & \text { if } t \leq \tau(T, x) .\end{cases}
$$

Given $\varepsilon>0$ such that $0<\tau(t, x)-\varepsilon<\tau(t, x)<\tau(t, x)+\varepsilon<\tau_{*}(t, T, x)$, there exist $t_{1}, t_{2} \in(0, T)$ such that $x=\varphi\left(t_{1} ; \tau(t, x)-\varepsilon, 0\right)=\varphi\left(t_{2} ; \tau(t, x)+\right.$ $\varepsilon, 0)$. Taking $\delta>0$ such that $\delta:=\min \left\{t-t_{1}, t_{2}-t\right\}$, it is shown that $|t-\hat{t}|<\delta$ implies $|\tau(t, x)-\tau(\hat{t}, x)|<\varepsilon$. When $x=z_{0}(t)$ or $x=0$, it is easily checked that $\tau(\cdot, x)$ is right or left continuous respectively by the same way.

Next, we show that $\tau$ is continuous on $U$. Let $(t, x) \in U$ and let $t_{n} \rightarrow t$ and $x_{n} \rightarrow x$. We may assume that $t_{n} \neq t$. Then there is a subsequence $t_{n_{k}}$ such that $t_{n_{k}} \uparrow t$ or $t_{n_{k}} \downarrow t$. We consider the former case. For the latter case, the same fact holds. Take $b>0$ such as $0 \leq x<b<z_{0}(t)$. Then for each $y \in[0, b], k \mapsto \tau\left(t_{n_{k}}, y\right)$ is increasing and $\lim _{k \rightarrow \infty} \tau\left(t_{n_{k}}, y\right)=\tau(t, y)$ as shown above. Further, $y \mapsto \tau\left(t_{n_{k}}, y\right.$ ) (for each sufficiently large $k$ ) and $y \mapsto \tau(t, y)$ are continuous by (i). Hence by Dini's theorem, we have $\lim _{k \rightarrow \infty} \tau\left(t_{n_{k}}, y\right)=\tau(t, y)$ uniformly for $y \in[0, b]$. Therefore, we conclude that $\lim _{k \rightarrow \infty} \tau\left(t_{n_{k}}, x_{n_{k}}\right)=\tau(t, x)$. Since the limit is common for the subsequences, we establish the continuity of $(t, x) \mapsto \tau(t, x)$.

The next lemma shows some differentiability properties of the characteristics with respect to the second and third arguments, and they are needed for changes of variables we will use often later.

Lemma 3.4. Let $x=\varphi(t ; \tau, \eta)$.

(i) $x$ is differentiable with respect to $\tau$ and

$$
\frac{d x}{d \tau}=-V(\eta, \tau) \exp \left(\int_{\tau}^{t} V_{x}(\varphi(\sigma ; \tau, \eta), \sigma) d \sigma\right) .
$$

(ii) $x$ is differentiable with respect to $\eta$ and

$$
\frac{d x}{d \eta}=\exp \left(\int_{\tau}^{t} V_{x}(\varphi(\sigma ; \tau, \eta), \sigma) d \sigma\right)
$$

Proof. (i) By Lemma 3.2 (ii), the function $\tau \mapsto \varphi(t ; \tau, \eta)$ is differentiable almost everywhere. On the other hand, invoking (3.1) and the Lebesgue 
bounded convergence theorem, we find that

$$
\begin{aligned}
\frac{1}{h}[\varphi(t ; \tau+h, \eta)-\varphi(t ; \tau, \eta)] & \\
= & \frac{1}{h}\left[\int_{\tau+h}^{t} V(\varphi(\sigma ; \tau+h, \eta), \sigma) d \sigma-\int_{\tau}^{t} V(\varphi(\sigma ; \tau, \eta), \sigma) d \sigma\right] \\
= & -\frac{1}{h} \int_{\tau}^{\tau+h} V(\varphi(\sigma ; \tau+h, \eta), \sigma) d \sigma \\
& +\int_{\tau}^{t} \frac{1}{h}[V(\varphi(\sigma ; \tau+h, \eta), \sigma)-V(\varphi(\sigma ; \tau, \eta), \sigma)] d \sigma \\
& \rightarrow-V(\varphi(\tau ; \tau, \eta), \tau)+\int_{\tau}^{t} V_{x}(\varphi(\sigma ; \tau, \eta), \sigma) \frac{\partial}{\partial \tau} \varphi(\sigma ; \tau, \eta) d \sigma \quad \text { as } h \rightarrow 0 .
\end{aligned}
$$

Therefore

$$
\frac{d x}{d \tau}=\frac{\partial}{\partial \tau} \varphi(t ; \tau, \eta)=-V(\eta, \tau) \exp \left(\int_{\tau}^{t} V_{x}(\varphi(\sigma ; \tau, \eta), \sigma) d \sigma\right) .
$$

(ii) Similarly to (i), one can show that (ii) holds.

Now we give some continuity properties of $L^{1}$-functions along the characteristics with respect to the $L^{1}$-norm.

Lemma 3.5. Let $f \in L^{1}:=L^{1}(0, l)$ and $0 \leq s<t$. Then we have

$$
\begin{aligned}
& \int_{0}^{l}|f(\eta)-f(\varphi(s ; t, \varphi(\hat{t} ; s, \eta)))| d \eta \rightarrow 0 \quad \text { as } \hat{t} \downarrow t \\
& \int_{0}^{l}|f(\eta)-f(\varphi(s ; \hat{t}, \varphi(t ; s, \eta)))| d \eta \rightarrow 0 \quad \text { as } \hat{t} \uparrow t .
\end{aligned}
$$

Proof. We will show only (3.3) because (3.4) is similar. For any $\varepsilon>0$, there exists an $\hat{f} \in C_{0}(0, l)$ such that $\|f-\hat{f}\|_{L^{1}}<\varepsilon$. Here $C_{0}(0, l)$ is the space of continuous functions having compact support in $(0, l)$. Then, we have

$$
\begin{aligned}
& \int_{0}^{l}|f(\eta)-f(\varphi(s ; t, \varphi(\hat{t} ; s, \eta)))| d \eta \\
& \leq \int_{0}^{l}|f(\eta)-\hat{f}(\eta)| d \eta+\int_{0}^{l}|\hat{f}(\eta)-\hat{f}(\varphi(s ; t, \varphi(\hat{t} ; s, \eta)))| d \eta \\
& \quad+\int_{0}^{l}|\hat{f}(\varphi(s ; t, \varphi(\hat{t} ; s, \eta)))-f(\varphi(s ; t, \varphi(\hat{t} ; s, \eta)))| d \eta .
\end{aligned}
$$

By Lemma 3.2 (ii), for each $\eta$ and $s,|\eta-\varphi(s ; \hat{t}, \varphi(t ; \hat{s}, \eta))| \rightarrow 0$ as $\hat{t} \downarrow t$. Therefore, by the Lebesgue bounded convergence theorem, we have

$$
\int_{0}^{l}|\hat{f}(\eta)-\hat{f}(\varphi(s ; \hat{t}, \varphi(t ; s, \eta)))| d \eta \rightarrow 0 \quad \text { as } \hat{t} \downarrow t .
$$


In order to estimate the third term, put $\lambda:=\varphi(s ; t, \varphi(\hat{t} ; s, \eta))$. Then by $(2.4)$

$$
\eta=\varphi(s ; \hat{t}, \varphi(t ; s, \lambda))=\varphi(t ; s, \lambda)+\int_{\hat{t}}^{s} V(\varphi(\sigma ; \hat{t}, \varphi(t ; s, \lambda), \sigma) d \sigma .
$$

Hence

$$
\begin{aligned}
\frac{d \eta}{d \lambda} & =\frac{\partial}{\partial \lambda} \varphi(s ; \hat{t}, \varphi(t ; s, \lambda)) \\
& =\frac{\partial}{\partial \lambda} \varphi(\hat{t} ; s, \lambda)+\int_{\hat{t}}^{s} V_{x}\left(\varphi(\sigma ; \hat{t}, \varphi(t ; s, \lambda), \sigma) \frac{\partial}{\partial \lambda} \varphi(\sigma ; \hat{t}, \varphi(t ; s, \lambda)) d \sigma\right.
\end{aligned}
$$

from which we obtain

$$
\frac{d \eta}{d \lambda}=\frac{\partial}{\partial \lambda} \varphi(t ; s, \lambda) \exp \left(\int_{\hat{t}}^{s} V_{x}(\varphi(\sigma ; \hat{t}, \varphi(t ; s, \lambda)), \sigma) d \sigma\right) .
$$

On the other hand, since $\varphi(t ; s, \lambda)=\lambda+\int_{s}^{t} V(\varphi(\sigma ; s, \lambda), \sigma) d \sigma$, we have

$$
\frac{\partial}{\partial \lambda} \varphi(\hat{t} ; s, \lambda)=1+\int_{s}^{\hat{t}} V_{x}(\varphi(\sigma ; s, \lambda), \sigma) \frac{\partial}{\partial \lambda} \varphi(\sigma ; s, \lambda) d \sigma
$$

which yields

$$
\frac{\partial}{\partial \lambda} \varphi(t ; s, \lambda)=\exp \left(\int_{s}^{t} V_{x}(\varphi(\sigma ; s, \lambda), \sigma) d \sigma\right) .
$$

Thus, we have

$$
\frac{d \eta}{d \lambda}=\exp \left(\int_{s}^{t} V_{x}(\varphi(\sigma ; s, \lambda), \sigma) d \sigma\right) \exp \left(\int_{\hat{t}}^{s} V_{x}(\varphi(\sigma ; s, \varphi(t ; s, \lambda)), \sigma) d \sigma\right) .
$$

Accordingly, we get the following estimate.

$$
\begin{aligned}
& \int_{0}^{l}|f(\eta)-f(\varphi(s ; t, \varphi(\hat{t} ; s, \eta)))| d \eta \\
& \quad \leq\|f-\hat{f}\|_{L^{1}}+\int_{0}^{l}|\hat{f}(\eta)-\hat{f}(\varphi(s ; t, \varphi(\hat{t} ; s, \eta)))| d \eta \\
& \quad+e^{2 L_{V} T} \int_{\varphi(s ; t, \varphi(\hat{t} ; s, 0))}^{l}|\hat{f}(\lambda)-f(\lambda)| d \lambda \\
& \quad \leq\left(1+e^{2 L_{V} T}\right)\|f-\hat{f}\|_{L^{1}}+\int_{0}^{l}|\hat{f}(\eta)-\hat{f}(\varphi(s ; t, \varphi(\hat{t} ; s, \eta)))| d \eta .
\end{aligned}
$$

Taking the limit superior on both sides yields

$$
\begin{aligned}
\limsup _{\hat{t} \downarrow t} \int_{0}^{l}|f(\eta)-f(\varphi(s ; t, \varphi(\hat{t} ; s, \eta)))| d \eta & \leq\left(1+e^{2 L_{V} T}\right)\|f-\hat{f}\|_{L^{1}} \\
& \leq\left(1+e^{2 L_{V} T}\right) \varepsilon .
\end{aligned}
$$

This completes the proof. 


\section{Proof of Theorem 2.1}

Given $r>0$, take $u_{a} \in L^{1}$ such that $\left\|u_{a}\right\|_{L^{1}} \leq r$. Define

$$
M_{T}:=\left\{u \in L_{a, T} \mid u(\cdot, a)=u_{a}(\cdot) \text { and }\|u\|_{L_{a, T}} \leq 2 r\right\} .
$$

Obviously, $M_{T}$ is a closed subset of $L_{a, T}$ and so a complete metric space. Define a mapping $K$ on $M_{T}$ as follows: For $u \in M_{T}, t \in[a, T]$,

$$
\begin{aligned}
& K u(x, t):= \\
& \begin{cases}\frac{\tilde{F}(\tau, u(\cdot, \tau))}{V(0, \tau)}+\int_{\tau}^{t} \tilde{G}(s, u(\cdot, s))(\varphi(s ; \tau, 0)) d s & \text { a.e. } x \in\left(0, z_{a}(t)\right), \\
u_{a}(\varphi(a ; t, x))+\int_{a}^{t} \tilde{G}(s, u(\cdot, s))(\varphi(s ; t, x)) d s & \text { a.e. } x \in\left(z_{a}(t), l\right),\end{cases}
\end{aligned}
$$

where $\tau:=\tau(t, x)$ is the one defined by (2.2), $\tilde{F}$ and $\tilde{G}$ are defined by (2.6) and (2.7) respectively in Section 2 .

We will seek the fixed point of the mapping $K$. For that purpose, we will show that $K$ maps $M_{T}$ into itself and that $K$ is contractive for some $T>a$.

Step 1: First, we show that $K: M_{T} \rightarrow M_{T}$ for $T=a+\delta$ with small $\delta>0$.

(i) For $u \in M_{T}, t \in[a, T]$,

$$
\begin{aligned}
& \int_{0}^{l}|K u(x, t)| d x \leq \int_{0}^{z_{a}(t)}\left|\frac{\tilde{F}(\tau, u(\cdot, \tau))}{V(0, \tau)}\right| d x \\
& \quad+\int_{0}^{z_{a}(t)} \int_{\tau}^{t}|\tilde{G}(s, u(\cdot, s))(\varphi(s ; \tau, 0))| d s d x \\
& \quad+\int_{z_{a}(t)}^{l}\left|u_{a}(\varphi(a ; t, x))\right| d x+\int_{z_{a}(t)}^{l} \int_{a}^{t}|\tilde{G}(s, u(\cdot, s))(\varphi(s ; t, x))| d s d x \\
& =: I_{1}+I_{2}+I_{3}+I_{4} .
\end{aligned}
$$

By Lemma 3.4 (i) and (F), we have

$$
\begin{aligned}
I_{1} & =\int_{a}^{t}\left|\frac{\tilde{F}(\tau, u(\cdot, \tau))}{V(0, \tau)}\right| V(0, \tau) \exp \left(\int_{\tau}^{t} V_{x}(\varphi(s ; \tau, 0), s) d s\right) d \tau \\
& \leq e^{L_{V}(T-a)}\left\{\int_{a}^{t}|C(\tau)| d \tau+\int_{a}^{t}|F(u(\cdot, \tau))| d \tau\right\} \\
& \leq e^{L_{V}(T-a)}\left\{C_{0}(t-a)+\int_{a}^{t}|F(u(\cdot, \tau))-F(0)| d \tau+\int_{a}^{t}|F(0)| d \tau\right\} \\
& \leq e^{L_{V}(T-a)}\left\{C_{0}(t-a)+c_{1}(2 r) \int_{a}^{t}\|u(\cdot, \tau)\|_{L^{1}} d \tau+(t-a)|F(0)|\right\} \\
& \leq e^{L_{V}(T-a)}\left[C_{0}+2 r \cdot c_{1}(2 r)+|F(0)|\right](T-a),
\end{aligned}
$$

where $C_{0}:=\sup _{t \in[0, T]} C(t)$. 
For $I_{2}$ and $I_{4}$, use the change of variable $\eta=\varphi(s ; \tau, 0)=\varphi(s ; t, x)$. By Lemma 3.4 (ii), we obtain

$$
\begin{aligned}
I_{2}+I_{4} \leq & e^{L_{V}(T-a)}\left\{\int_{a}^{t} \int_{0}^{z_{a}(s)}|\tilde{G}(s, u(\cdot, s))(\eta)| d \eta d s\right. \\
& \left.+\int_{a}^{t} \int_{z_{a}(s)}^{l}|\tilde{G}(s, u(\cdot, s))(\eta)| d \eta d s\right\} \\
\leq & e^{L_{V}(T-a)}\left\{\int_{a}^{t} \int_{0}^{l}|G(u(\cdot, s))(\eta)| d \eta d s\right. \\
& \left.+\int_{a}^{t} \int_{0}^{l}\left|V_{x}(\eta, s) u(\eta, s)\right| d x d s\right\} .
\end{aligned}
$$

By $(\mathrm{G})$ and $(\mathrm{V})$, we have

$$
\begin{gathered}
\int_{0}^{l}|G(u(\cdot, s))(\eta)| d \eta \leq\|G(u(\cdot, s))-G(0)\|_{L^{1}}+\|G(0)\|_{L^{1}} \\
\leq c_{2}(2 r)\|u(\cdot, s)\|_{L^{1}}+\|G(0)\|_{L^{1}} \leq c_{2}(2 r) \cdot 2 r+\|G(0)\|_{L^{1}} \\
\int_{0}^{l}\left|V_{x}(\eta, s) u(\eta, s)\right| d \eta \leq L_{V}\|u(\cdot, s)\|_{L^{1}} \leq L_{V} \cdot 2 r .
\end{gathered}
$$

Therefore, we get the following inequality

$$
I_{2}+I_{4} \leq e^{L_{V}(T-a)}\left[\left(c_{2}(2 r)+L_{V}\right) \cdot 2 r+\|G(0)\|_{L^{1}}\right](T-a) .
$$

For $I_{3}$, the change of variable $\xi=\varphi(a ; t, x)$ leads to

$$
I_{3} \leq e^{L_{V}(T-a)} \int_{a}^{l}\left|u_{a}(\xi)\right| d \xi \leq r e^{L_{V}(T-a)} .
$$

Consequently,

$$
\begin{aligned}
& I_{1}+I_{2}+I_{3}+I_{4} \\
& \leq e^{L_{V}(T-a)}\left[C_{0}+\left(c_{1}(2 r)+c_{2}(2 r)+L_{V}\right) \cdot 2 r\right. \\
& \left.\quad+|F(0)|+\|G(0)\|_{L^{1}}\right](T-a)+r e^{L_{V}(T-a)} .
\end{aligned}
$$

Choose $\delta>0$ so small that

$$
\begin{aligned}
e^{L_{V} \delta}\left[C_{0}\right. & +\left(c_{1}(2 r)+c_{2}(2 r)+L_{V}\right) \cdot 2 r \\
& \left.+|F(0)|+\|G(0)\|_{L^{1}}\right] \delta+r e^{L_{V} \delta} \leq 2 r .
\end{aligned}
$$

Then combining (4.2) with (4.5), we have $\sup _{a \leq t \leq T}\|K u(\cdot, t)\|_{L^{1}} \leq 2 r$ for $T=a+\delta$. 
(ii) (Continuity of $t \mapsto K u(\cdot, t))$ Let $u \in M_{T}$ and $t \in[a, T]$. We will show only the right-continuity. The left-continuity is proved by exchanging $t$ and $\hat{t}$. We will just give some remarks on proving it below.

Let $a \leq t<\hat{t} \leq T$. From (4.1) we have

$$
\begin{aligned}
& \int_{0}^{l}|K u(x, t)-K u(x, \hat{t})| d x \\
& \leq \int_{0}^{z_{a}(t)}\left|\frac{\tilde{F}(\tau, u(\cdot, \tau))}{V(0, \tau)}-\frac{\tilde{F}(\hat{\tau}, u(\cdot, \hat{\tau}))}{V(0, \hat{\tau})}\right| d x \\
& \quad+\int_{0}^{z_{a}(t)}\left|\int_{\tau}^{t} \tilde{G}(s, u(\cdot, s))(\varphi(s ; \tau, 0)) d s-\int_{\hat{\tau}}^{\hat{t}} \tilde{G}(s, u(\cdot, s))(\varphi(s ; \hat{\tau}, 0)) d s\right| d x \\
& \quad+\int_{z_{a}(t)}^{z_{a}(\hat{t})} \mid u_{a}(\varphi(a ; t, x))+\int_{a}^{t} \tilde{G}(s, u(\cdot, s))(\varphi(s ; t, x)) d s \\
& \quad-\frac{\tilde{F}(\hat{\tau}, u(\cdot, \hat{\tau}))}{V(0, \hat{\tau})}-\int_{\hat{\tau}}^{\hat{t}} \tilde{G}(s, u(\cdot, s))(\varphi(s ; \hat{\tau}, 0)) d s \mid d x \\
& \quad+\int_{z_{a}(\hat{t})}^{l}\left|u_{a}(\varphi(a ; t, x))-u_{a}(\varphi(a ; \hat{t}, x))\right| d x \\
& \quad+\int_{z_{a}(\hat{t})}^{l}\left|\int_{a}^{t} \tilde{G}(s, u(\cdot, s))(\varphi(s ; t, x)) d s-\int_{a}^{\hat{t}} \tilde{G}(s, u(\cdot, s))(\varphi(s ; \hat{t}, x)) d s\right| d x \\
& =: J_{1}+J_{2}+J_{3}+J_{4}+J_{5},
\end{aligned}
$$

where $\tau:=\tau(t, x)$ and $\hat{\tau}:=\tau(\hat{t}, x)$.

First, consider $J_{1}$. For simplicity of notation, we put $B(t):=\tilde{F}(t, u(\cdot, t))$. Then

$$
J_{1} \leq \int_{0}^{z_{a}(t)} \frac{1}{V(0, \tau)}|B(\tau)-B(\hat{\tau})| d x+\int_{0}^{z_{a}(t)}\left|\frac{1}{V(0, \tau)}-\frac{1}{V(0, \hat{\tau})}\right||B(\hat{\tau})| d x .
$$

Using the change of variable $\xi=\tau=\tau(t, x)$, we obtain

$$
\begin{aligned}
J_{1} \leq & e^{L_{V} T} \int_{a}^{t}|B(\xi)-B(\tau(\hat{t}, \varphi(t ; \xi, 0)))| d \xi \\
& +e^{L_{V} T} \int_{a}^{t}\left|1-\frac{V(0, \xi)}{V(0, \tau(\hat{t}, \varphi(t ; \xi, 0)))}\right||B(\tau(\hat{t}, \varphi(t ; \xi, 0)))| d \xi \\
\leq & e^{L_{V} T} \int_{a}^{t}|B(\xi)-B(\tau(\hat{t}, \varphi(t ; \xi, 0)))| d \xi \\
& +e^{L_{V} T} \sup _{a \leq t \leq T}|B(t)| \frac{1}{V_{1}} \int_{a}^{t}|V(0, \tau(\hat{t}, \varphi(t ; \xi, 0)))-V(0, \xi)| d \xi
\end{aligned}
$$

where $V_{1}:=\min _{a \leq t \leq T} V(0, t)>0$. By virtue of Lemma 3.3 (ii), for each $\xi \in[a, t]$

$$
|\xi-\tau(\hat{t}, \varphi(t ; \xi, 0))|=|\tau(t, \varphi(t ; \xi, 0))-\tau(\hat{t}, \varphi(t ; \xi, 0))| \rightarrow 0 \quad \text { as } \hat{t} \downarrow t .
$$


Noting that $B(t)$ and $V(0, t)$ are continuous in $t$ and bounded on $[a, T]$, we have $J_{1} \rightarrow 0$ as $\hat{t} \downarrow t$ by the Lebesgue bounded convergence theorem.

Next, we shall estimate $J_{2}$ : We may assume $\varphi(\hat{t}, t, 0)<z_{a}(t)$ since $\hat{t}$ is colse enough to $t$. For simplicity, we put $G_{s}(x):=\tilde{G}(s, u(\cdot, s))(x)$. Then

$$
\begin{aligned}
J_{2} \leq & \int_{0}^{\varphi(\hat{t} ; t, 0)} \int_{\tau}^{t}\left|G_{s}(\varphi(s ; \tau, 0))\right| d s d x+\int_{0}^{\varphi(\hat{t} ; t, 0)} \int_{\hat{\tau}}^{\hat{t}}\left|G_{s}(\varphi(s ; \hat{t}, 0))\right| d s d x \\
& +\int_{\varphi(\hat{t} ; t, 0)}^{z_{a}(t)} \int_{\tau}^{\hat{\tau}}\left|G_{s}(\varphi(s ; \tau, 0))\right| d s d x+\int_{\varphi(\hat{t} ; t, 0)}^{z_{a}(t)} \int_{t}^{\hat{t}}\left|G_{s}(\varphi(s ; \hat{\tau}, 0))\right| d s d x \\
& +\int_{\varphi(\hat{t} ; t, 0)}^{z_{a}(t)}\left|\int_{\hat{\tau}}^{t}\right| G_{s}(\varphi(s ; \tau, 0))-G_{s}(\varphi(s ; \hat{\tau}, 0))|d s| d x \\
= & : J_{21}+J_{22}+J_{23}+J_{24}+J_{25} .
\end{aligned}
$$

For simplicity of notation, we put $\tau(x)=\tau(t, x)$ and $\hat{\tau}(x)=\tau(\hat{t}, x)$. Using Fubini's theorem and the change of variable $\eta=\varphi(s ; \tau, 0)=\varphi(s ; t, x)$ together with Lemma 3.4, we have

$$
\begin{aligned}
J_{21} & =\int_{\tau(\varphi(\hat{t} ; t, 0))}^{t} \int_{\tau^{-1}(s)}^{\varphi(\hat{t} ; t, 0)}\left|G_{s}(\varphi(s ; \tau, 0))\right| d x d s \\
& =\int_{\tau(\varphi(\hat{t} ; t, 0))}^{t} \int_{0}^{\varphi(s ; t, \varphi(\hat{t} ; t, 0))}\left|G_{s}(\eta)\right| \exp \left(\int_{s}^{t} V_{x}(\varphi(\sigma ; s, \eta), \sigma) d \sigma\right) d \eta d s \\
& \leq e^{L_{V} T} \sup _{a \leq s \leq T}\left\|G_{s}\right\|_{L^{1}}[t-\tau(\varphi(\hat{t} ; t, 0))] .
\end{aligned}
$$

Since $\tau(t, \varphi(\hat{t} ; t, 0)) \rightarrow t$ as $\hat{t} \downarrow t$, we obtain $J_{21} \rightarrow 0$.

Similarly, we have

$$
\begin{aligned}
J_{22} & =\int_{t}^{\hat{t}} \int_{0}^{\varphi(s ; t, 0)}\left|G_{s}(\eta)\right| \exp \left(\int_{s}^{\hat{t}} V_{x}(\varphi(\sigma ; s, \eta), \sigma) d \sigma\right) d \eta d s \\
& \leq e^{L_{V} T} \sup _{a \leq s \leq T}\left\|G_{s}\right\|_{L^{1}}(\hat{t}-t) .
\end{aligned}
$$

Thus $J_{22} \rightarrow 0$ as $\hat{t} \downarrow t$.

Consider $J_{23}$. We may assume $\hat{\tau}\left(z_{a}(t)\right)<\tau(\varphi(\hat{t} ; t, 0))$. Since we have 
$t=\hat{\tau}(\varphi(\hat{t} ; t, 0))>\tau(\varphi(\hat{t} ; t, 0))$, we obtain

$$
\begin{aligned}
J_{23}= & \int_{\tau(\varphi(\hat{t} ; t, 0))}^{t} \int_{\varphi(\hat{t} ; t, 0)}^{\hat{\tau}^{-1}(s)}\left|G_{s}(\varphi(s ; \tau, 0))\right| d x d s \\
& +\int_{\hat{\tau}\left(z_{a}(t)\right)}^{\tau(\varphi(\hat{t} ; t, 0))} \int_{\tau^{-1}(s)}^{\hat{\tau}^{-1}(s)}\left|G_{s}(\varphi(s ; \tau, 0))\right| d x d s \\
& +\int_{a}^{\hat{\tau}\left(z_{a}(t)\right)} \int_{\tau^{-1}(s)}^{z_{a}(t)}\left|G_{s}(\varphi(s ; \tau, 0))\right| d x d s \\
\leq & e^{L_{V} T} \int_{\tau(\varphi(\hat{t} ; t, 0))}^{t} \int_{\varphi(s ; t, \varphi(\hat{t} ; t, 0))}^{\varphi(s ; t, \varphi(\hat{t} ; s, 0))}\left|G_{s}(\eta)\right| d \eta d s \\
& +e^{L_{V} T} \int_{\hat{\tau}\left(z_{a}(t)\right)}^{\tau(\varphi(\hat{t} ; t, 0))} \int_{0}^{\varphi(s ; t, \varphi(\hat{t} ; s, 0))}\left|G_{s}(\eta)\right| d \eta d s \\
& +e^{L_{V} T} \int_{a}^{\hat{\tau}\left(z_{a}(t)\right)} \int_{0}^{z_{a}(s)}\left|G_{s}(\eta)\right| d \eta d s \\
\leq & e^{L_{V} T} \sup _{a \leq s \leq T}\left\|G_{s}\right\|_{L^{1}}\left[t-\tau(\varphi(\hat{t} ; t, 0))+\hat{\tau}\left(z_{a}(t)\right)-a\right] \\
& +e^{L_{V} T} \int_{\hat{\tau}\left(z_{a}(t)\right)}^{\tau(\varphi(\hat{t} ; t, 0))} \int_{0}^{\varphi(s ; t, \varphi(\hat{t}, s, 0))}\left|G_{s}(\eta)\right| d \eta d s .
\end{aligned}
$$

The first term tends to 0 since $\tau(\varphi(\hat{t} ; t, 0)) \rightarrow \tau(\varphi(t ; t, 0))=\tau(0)=t$ and $\hat{\tau}\left(z_{a}(t)\right) \rightarrow \tau\left(z_{a}(t)\right)=a$. From the fact that $\int_{0}^{\varphi(s ; t, \varphi(\hat{t} ; s, 0))}\left|G_{s}(\eta)\right| d \eta$ converges to 0 as $\hat{t} \downarrow t$ and bounded by $\sup _{a \leq s \leq T}\left\|G_{s}\right\|_{L^{1}}$, we find that the second term converges to 0 by the Lebesgue bounded convergence theorem. Hence $J_{23} \rightarrow 0$.

For $J_{24}$, we proceed very similarly to the case $J_{22}$ and obtain

$$
J_{24} \leq e^{L_{V} T} \int_{t}^{\hat{t}} \int_{\varphi(s ; t, 0)}^{\varphi\left(s, \hat{t}, z_{a}(t)\right)}\left|G_{s}(\eta)\right| d \eta d s \leq e^{L_{V} T} \sup _{a \leq s \leq T}\left\|G_{s}\right\|_{L^{1}}(\hat{t}-t) .
$$

Thus $J_{24} \rightarrow 0$.

Next we estimate $J_{25}$. As before, using Fubini's theorem and then by changing varuable $\eta=\varphi(s ; \tau, 0)=\varphi(s ; \hat{t}, x)$, we have

$$
\begin{aligned}
J_{25} & \leq e^{L_{V} T} \int_{\hat{\tau}\left(z_{a}(t)\right)}^{t} \int_{0}^{\varphi\left(s ; \hat{t}, z_{a}(t)\right)}\left|G_{s}(\varphi(s ; t, \varphi(\hat{t} ; s, \eta)))-G_{s}(\eta)\right| d \eta d s \\
& \leq e^{L_{V} T} \int_{a}^{t} \int_{0}^{l}\left|G_{s}(\varphi(s ; t, \varphi(\hat{t} ; s, \eta)))-G_{s}(\eta)\right| d \eta d s .
\end{aligned}
$$

By Lemma 3.5, we have

$$
\lim _{\hat{t} \downarrow t} \int_{0}^{l}\left|G_{s}(\varphi(s ; t, \varphi(\hat{t} ; s, \eta)))-G_{s}(\eta)\right| d \eta=0 .
$$


Also we find from Lemma 3.4 (see the proof of Lemma 3.5) that

$$
\int_{0}^{l}\left|G_{s}(\varphi(s ; t, \varphi(\hat{t} ; s, \eta)))-G_{s}(\eta)\right| d \eta \leq\left(e^{2 L_{V} T}+1\right) \sup _{a \leq s \leq T}\left\|G_{s}\right\|_{L^{1}} .
$$

Thus the Lebesgue bounded convergence theorem yields $J_{25} \rightarrow 0$.

Next consider $J_{3}$.

$$
\begin{aligned}
J_{3} \leq & \int_{z_{a}(t)}^{z_{a}(\hat{t})}\left|u_{a}(\varphi(a ; t, x))\right| d x+\int_{z_{a}(t)}^{z_{a}(\hat{t})}\left|\frac{B(\hat{\tau})}{V(0, \hat{\tau})}\right| d x \\
& +\int_{z_{a}(t)}^{z_{a}(\hat{t})} \int_{a}^{t}\left|G_{s}(\varphi(s ; t, x))\right| d s d x+\int_{z_{a}(t)}^{z_{a}(\hat{t})} \int_{\hat{\tau}}^{\hat{t}}\left|G_{s}(\varphi(s ; \hat{\tau}, 0))\right| d s d x \\
= & : J_{31}+J_{32}+J_{33}+J_{34} .
\end{aligned}
$$

It is easily seen that

$$
\begin{aligned}
& J_{31} \leq e^{L_{V} T} \int_{a}^{\varphi(a ; t, z(\hat{t}))}\left|u_{a}(\eta)\right| d \eta \\
& J_{32} \leq e^{L_{V} T} \int_{a}^{\tau\left(\hat{t}, z_{a}(t)\right)}|B(\xi)| d \xi \\
& J_{33} \leq e^{L_{V} T} \int_{a}^{t} \int_{z_{a}(s)}^{\varphi\left(s ; t, z_{a}(\hat{t})\right)}\left|G_{s}(\eta)\right| d \eta d s .
\end{aligned}
$$

Thus we find that $J_{31}+J_{32}+J_{33} \rightarrow 0$.

To estimate $J_{34}$ we may assume that $\hat{\tau}\left(z_{a}(t)\right)<t$. Then

$$
\begin{aligned}
J_{34} \leq & e^{L_{V} T} \int_{\hat{\tau}\left(z_{a}(t)\right)}^{\hat{t}} \int_{\varphi\left(s ; \hat{t}, z_{a}(t)\right)}^{z_{a}(s)}\left|G_{s}(\eta)\right| d \eta d s \\
& +e^{L_{V} T} \int_{a}^{\hat{\tau}\left(z_{a}(t)\right)} \int_{0}^{z_{a}(s)}\left|G_{s}(\eta)\right| d \eta d s \\
\leq & e^{L_{V} T} \int_{\hat{\tau}\left(z_{a}(t)\right)}^{\hat{t}} \int_{\varphi\left(s ; \hat{t}, z_{a}(t)\right)}^{z_{a}(s)}\left|G_{s}(\eta)\right| d \eta d s \\
& +e^{L_{V} T} \sup _{a \leq s \leq T}\left\|G_{s}\right\|_{L^{1}}[\hat{\tau}(z(t))-a] .
\end{aligned}
$$

By the Lebesgue bounded theorem, the first term tends to 0 as $\hat{t} \downarrow t$. The second term goes to 0 since $\hat{\tau}\left(z_{a}(t)\right) \rightarrow \tau\left(z_{a}(t)\right)=a$. Therefore $J_{34} \rightarrow 0$.

For $J_{4}$, one easily sees that

$$
J_{4} \leq e^{L_{V} T} \int_{0}^{l}\left|u_{a}(\varphi(a ; t, \varphi(\hat{t} ; a, \eta)))-u_{a}(\eta)\right| d \eta
$$


Thus by Lemma 3.5, we get $J_{4} \rightarrow 0$ as $\hat{t} \downarrow t$.

Finally $J_{5}$ is estimated as follows.

$$
\begin{aligned}
J_{5} \leq & \int_{a}^{t} \int_{z_{a}(\hat{t})}^{l}\left|G_{s}(\varphi(s ; t, x))-G_{s}(\varphi(s, \hat{t}, x))\right| d x d s \\
& +\int_{t}^{\hat{t}} \int_{z_{a}(\hat{t})}^{l} \mid G_{s}(\varphi(s ; \hat{t}, x) \mid d x d s \\
\leq & e^{L_{V} T} \int_{a}^{t} \int_{z_{a}(s)}^{l}\left|G_{s}(\varphi(s ; t, \varphi(\hat{t}, s, \eta)))-G_{s}(\eta)\right| d \eta d s \\
& +e^{L_{V} T} \int_{t}^{\hat{t}} \int_{z_{a}(s)}^{l}\left|G_{s}(\eta)\right| d \eta d s \\
\leq & e^{L_{V} T} \int_{a}^{t} \int_{0}^{l}\left|G_{s}(\varphi(s ; t, \varphi(\hat{t}, s, \eta)))-G_{s}(\eta)\right| d \eta d s \\
& +e^{L_{V} T} \sup _{a \leq s \leq T}\left\|G_{s}\right\|_{L^{1}}(\hat{t}-t) .
\end{aligned}
$$

By Lemma 3.5, we find that $J_{5} \rightarrow 0$ as $\hat{t} \downarrow t$.

Consequently, the right-continuity has been shown. To prove the leftcontinuity, let $\hat{t}<t$. Then by exchanging $t$ and $\hat{t}$, we obtain all the estimates above with $t$ and $\hat{t}$ exchanged. By the continuity of $\tau$ obtained in Lemma 3.3 (ii), we find that all the terms tends to 0 as $\hat{t} \uparrow t$. Hence the continuity is proved.

Step 2: We show that $K$ is a contraction mapping for $T=a+\delta$ with small $\delta>0$. For $u_{i} \in M_{T}(i=1,2)$, it follows from (4.1) that

$$
\begin{aligned}
\int_{0}^{l} \mid & K u_{1}(x, t)-K u_{2}(x, t) \mid d x \\
\leq & \int_{0}^{z_{a}(t)}\left|\frac{F\left(u_{1}(\cdot, \tau)\right)-F\left(u_{2}(\cdot, \tau)\right)}{V(0, \tau)}\right| d x \\
& +\int_{0}^{z_{a}(t)} \int_{\tau}^{t}\left|\tilde{G}\left(s, u_{1}(\cdot, s)\right)(\varphi(s ; \tau, 0))-\tilde{G}\left(s, u_{2}(\cdot, s)\right)(\varphi(s ; \tau, 0))\right| d s d x \\
& +\int_{z_{a}(t)}^{l} \int_{a}^{t}\left|\tilde{G}\left(s, u_{1}(\cdot, s)\right)(\varphi(s ; t, x))-\tilde{G}\left(s, u_{2}(\cdot, s)\right)(\varphi(s ; t, x))\right| d s d x \\
= & : P_{1}+P_{2}+P_{3} .
\end{aligned}
$$

By Lemma 3.4 (i) and (F),

$$
\begin{aligned}
P_{1} & \leq e^{L_{V}(T-a)} \int_{a}^{t}\left|F\left(u_{1}(\cdot, \tau)\right)-F\left(u_{2}(\cdot, \tau)\right)\right| d \tau \\
& \leq e^{L_{V}(T-a)} c_{1}(2 r) \int_{a}^{t}\left\|u_{1}(\cdot, \tau)-u_{2}(\cdot, \tau)\right\|_{L^{1}} d \tau \\
& \leq e^{L_{V}(T-a)} c_{1}(2 r)(T-a)\left\|u_{1}-u_{2}\right\|_{L_{a, T}} .
\end{aligned}
$$


By using the change of variable $\eta=\varphi(s ; t, x)=\varphi(s ; \tau, 0)$, and by $(\mathrm{G})$, the following estimate for $P_{2}+P_{3}$ holds:

$$
\begin{aligned}
& P_{2}+P_{3}= \\
& \int_{a}^{t} \int_{\tau_{t}-1}^{z_{a}(t)}\left|\tilde{G}\left(s, u_{1}(\cdot, s)\right)(\varphi(s ; \tau, 0))-\tilde{G}\left(s, u_{2}(\cdot, s)\right)(\varphi(s ; \tau, 0))\right| d x d s \\
& \quad+\int_{z_{a}(t)}^{l} \int_{a}^{t}\left|\tilde{G}\left(s, u_{1}(\cdot, s)\right)(\varphi(s ; t, x))-\tilde{G}\left(s, u_{2}(\cdot, s)\right)(\varphi(s ; t, x))\right| d x d s \\
& \leq e^{L_{V}(T-a)} \int_{a}^{t} \int_{0}^{z_{a}(s)}\left|\tilde{G}\left(s, u_{1}(\cdot, s)\right)(\eta)-\tilde{G}\left(s, u_{2}(\cdot, s)\right)(\eta)\right| d \eta d s \\
& \quad+e^{L_{V}(T-a)} \int_{a}^{t} \int_{z_{a}(s)}^{l}\left|\tilde{G}\left(s, u_{1}(\cdot, s)\right)(\eta)-\tilde{G}\left(s, u_{2}(\cdot, s)\right)(\eta)\right| d \eta d s \\
& =e^{L_{V}(T-a)} \int_{a}^{t} \int_{0}^{l}\left|\tilde{G}\left(s, u_{1}(\cdot, s)\right)(\eta)-\tilde{G}\left(s, u_{2}(\cdot, s)\right)(\eta)\right| d \eta d s \\
& \leq e^{L_{V}(T-a)} \int_{a}^{t}\left[\left\|G\left(u_{1}(\cdot, s)\right)-G\left(u_{2}(\cdot, s)\right)\right\|_{L^{1}}+L_{V}\left\|u_{1}(\cdot, s)-u_{2}(\cdot, s)\right\|_{L^{1}}\right] d s \\
& \leq e^{L_{V}(T-a)} \int_{a}^{t}\left[\left(c_{2}(2 r)+L_{V}\right)\left\|u_{1}(\cdot, s)-u_{2}(\cdot, s)\right\|_{L^{1}}\right] d s \\
& \leq e^{L_{V}(T-a)}\left(c_{2}(2 r)+L_{V}\right)(T-a)\left\|u_{1}-u_{2}\right\|_{L_{a}, T} \cdot
\end{aligned}
$$

Accordingly,

$$
\left\|K u_{1}-K u_{2}\right\|_{L_{a, T}} \leq e^{L_{V}(T-a)}\left[c_{1}(2 r)+c_{2}(2 r)+L_{V}\right](T-a)\left\|u_{1}-u_{2}\right\|_{L_{a, T}} .
$$

Therefore if we choose $\delta>0$ so small that

$$
e^{L_{V} \delta}\left[c_{1}(2 r)+c_{2}(2 r)+L_{V}\right] \delta<1
$$

then $K$ becomes a contraction on $M_{T}$ with $T=a+\delta$.

Consequently, by the contraction mapping theorem, there is a unique fixed point $u \in M_{T}$ such that $K u=u$. This completes the proof.

\section{Proof of Theorem 2.2}

The proof is similar to the proof of the mapping $\mathrm{K}$ to be contractive as done in the previous section. We need a little more careful estimation.

For the solutions $u, \hat{u} \in L_{a, T}$ of (SDP) with initial data $u_{a}, \hat{u}_{a}$ respec- 
tively, it follows from (2.5) that

$$
\begin{aligned}
& \|u(\cdot, t)-\hat{u}(\cdot, t)\|_{L^{1}} \leq \int_{0}^{z_{a}(t)}\left|\frac{F(u(\cdot, \tau))-F(\hat{u}(\cdot, \tau))}{V(0, \tau)}\right| d x \\
& \quad+\int_{0}^{z_{a}(t)} \int_{\tau}^{t}|\tilde{G}(s, u(\cdot, s))(\varphi(s ; \tau, 0))-\tilde{G}(s, \hat{u}(\cdot, s))(\varphi(s ; \tau, 0))| d s d x \\
& \quad+\int_{z_{a}(t)}^{l} \int_{a}^{t}\left|u_{a}(\varphi(a ; t, x))-\hat{u}_{a}(\varphi(a ; t, x))\right| d x \\
& \quad+\int_{z_{a}(t)}^{l} \int_{a}^{t}|\tilde{G}(s, u(\cdot, s))(\varphi(s ; t, x))-\tilde{G}(s, \hat{u}(\cdot, s))(\varphi(s ; t, x))| d s d x \\
& =: R_{1}+R_{2}+R_{3}+R_{4} .
\end{aligned}
$$

By changing variable $\tau:=\tau(t, x)$ it follows from Lemma 3.4 (i) and (F) that

$$
\begin{aligned}
R_{1} & \leq \int_{a}^{t} e^{L_{V}(t-\tau)}|F(u(\cdot, \tau))-F(\hat{u}(\cdot, \tau))| d \tau \\
& \leq c_{1}(r) \int_{a}^{t} e^{L_{V}(t-\tau)}\|u(\cdot, \tau)-\hat{u}(\cdot, \tau)\|_{L^{1}} d \tau .
\end{aligned}
$$

As the estimation of $I_{3}$ in the previous section,

$$
R_{3} \leq e^{L_{V}(t-a)} \int_{0}^{l}\left|u_{a}(\xi)-\hat{u}_{a}(\xi)\right| d \xi .
$$

Similarly to the estimate of $P_{2}+P_{3}$, by using the change of variable $\eta=$ $\varphi(s ; t, x)=\varphi(s ; \tau, 0)$, and by $(\mathrm{G})$, we have

$$
\begin{aligned}
R_{2} & +R_{4} \\
\leq & \int_{a}^{t} \int_{0}^{z_{a}(s)} e^{L_{V}(t-s)}|\tilde{G}(s, u(\cdot, s))(\eta)-\tilde{G}(s, \hat{u}(\cdot, s))(\eta)| d \eta d s \\
& +\int_{a}^{t} \int_{z_{a}(s)}^{l} e^{L_{V}(t-s)}|\tilde{G}(s, u(\cdot, s))(\eta)-\tilde{G}(s, \hat{u}(\cdot, s))(\eta)| d \eta d s \\
\leq & \int_{a}^{t} e^{L_{V}(t-s)}\left[\|G(u(\cdot, s))-G(\hat{u}(\cdot, s))\|_{L^{1}}+L_{V}\|u(\cdot, s)-\hat{u}(\cdot, s)\|_{L^{1}}\right] d s \\
\leq & \left(c_{2}(r)+L_{V}\right) \int_{a}^{t} e^{L_{V}(t-s)}\left[\|u(\cdot, s)-\hat{u}(\cdot, s)\|_{L^{1}}\right] d s .
\end{aligned}
$$

Therefore we obtain

$$
\begin{aligned}
& e^{-L_{V}(t-a)}\|u(\cdot, t)-\hat{u}(\cdot, t)\|_{L^{1}} \\
& \leq\left\|u_{a}-\hat{u}_{a}\right\|_{L^{1}}+\left[c_{1}(r)+c_{2}(r)+L_{V}\right] \int_{a}^{t} e^{-L_{V}(s-a)}\|u(\cdot, s)-\hat{u}(\cdot, s)\|_{L^{1}} d s .
\end{aligned}
$$

By Gronwall's lemma,

$e^{-L_{V}(t-a)}\|u(\cdot, t)-\hat{u}(\cdot, t)\|_{L^{1}} \leq \exp \left[\left(c_{1}(r)+c_{2}(r)+L_{V}\right)(t-a)\right]\left\|u_{a}-\hat{u}_{a}\right\|_{L^{1}}$, from which the desired estimate is established. 


\section{REFERENCES}

[1] A. Calsina and J. Saldaña, A model of physiologically structured population dynamics with a nonlinear individual growth rate, J. Math. Biol. 33 (1995), 335-364.

[2] Ph. Clément, H. J. A. M. Heijmans, et al., One-Parameter Semigroups, CWI Monograph \#5, North-Holland, Amsterdam, 1987.

[3] J. A. J. Metz and O. Diekmann (eds.), The Dynamics of Physiologically Structured Populations, Lect. Notes in Biomath. \#68, Springer-Verlag, Berlin, Heidelberg, 1986.

[4] G. F. Webb, Theory of Nonlinear Age-Dependent Population Dynamics, Marcel Dekker, New York, 1985.

Department of Mathematics

SHIMANE UNIVERSITY

MAtsue 690-8504, JAPAN

E-mail address: nkato@riko.shimane-u.ac.jp 


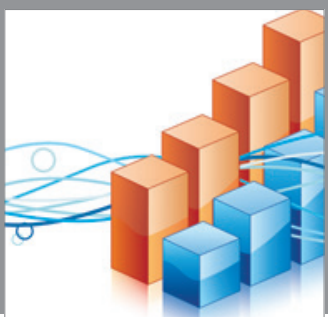

Advances in

Operations Research

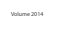

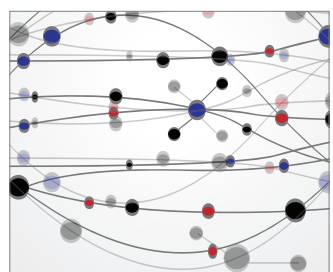

\section{The Scientific} World Journal
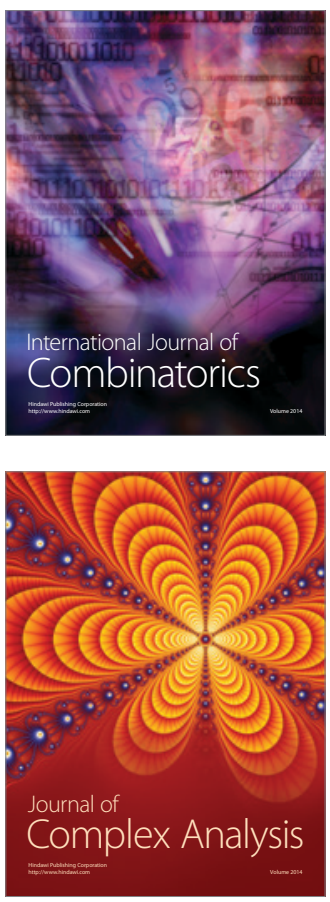

International Journal of

Mathematics and

Mathematical

Sciences
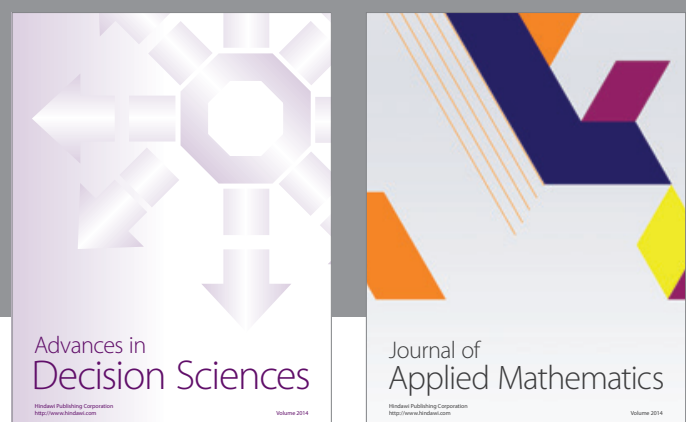

Journal of

Applied Mathematics
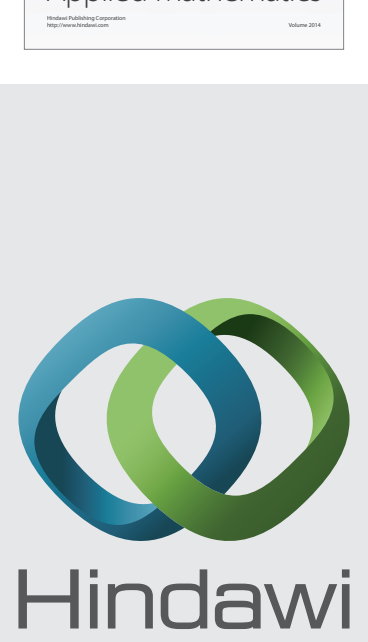

Submit your manuscripts at http://www.hindawi.com
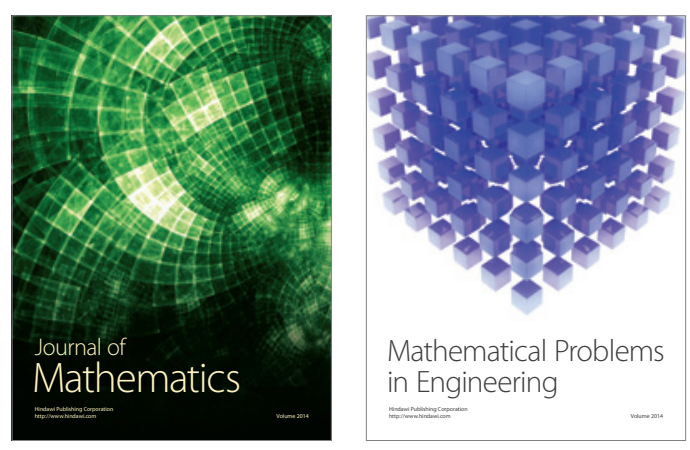

Mathematical Problems in Engineering
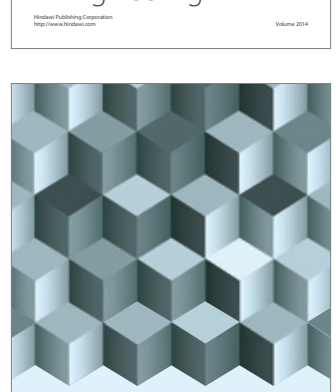

Journal of

Function Spaces
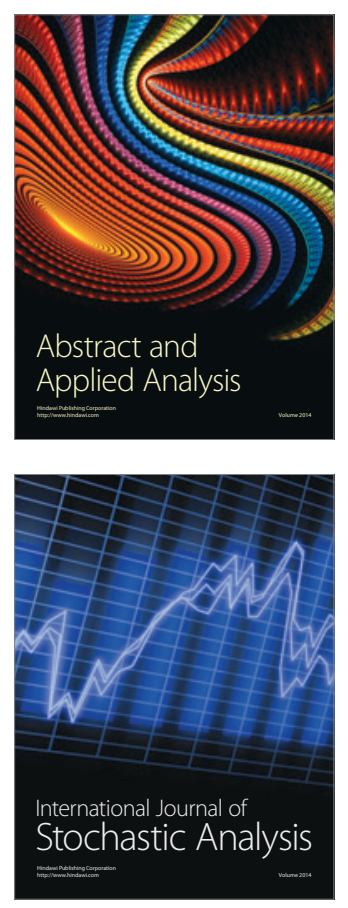

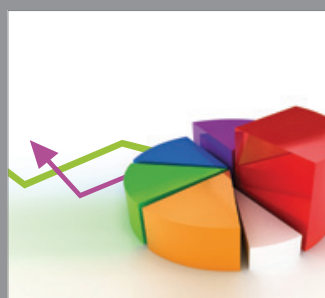

ournal of

Probability and Statistics

Promensencen
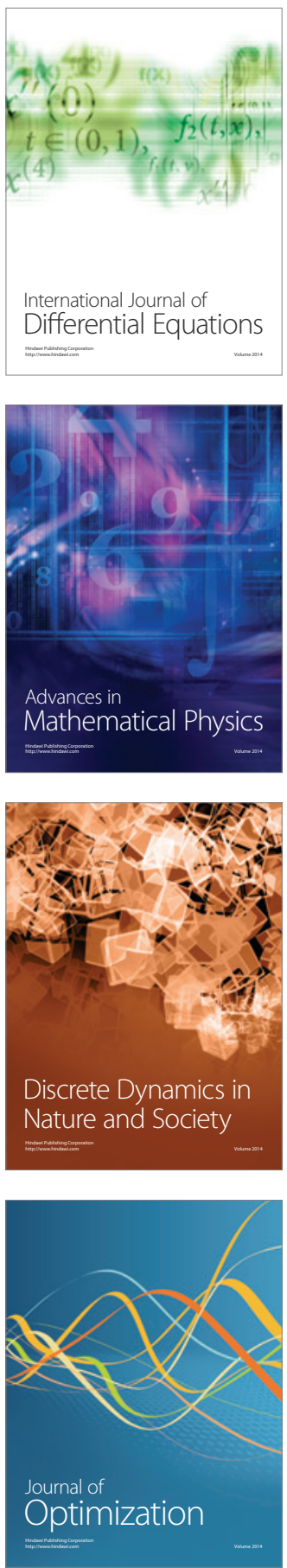